\title{
An Autopsy Case of Fulminant, Suppurative Bacterial Myocarditis Caused by Group B Streptococcus
}

\author{
Ryuta Nakashima ${ }^{1,2}$, Munenori Kotoku ${ }^{3}$, Ayako Gamachi ${ }^{4}$, Nobuhiro Inagaki ${ }^{2}$ and \\ Shunji Kasaoka ${ }^{5}$
}

\begin{abstract}
:
A 61-year-old woman was admitted to our hospital with a fever, nausea, diarrhea, and back pain. Her condition rapidly deteriorated, and she was transferred to the intensive-care unit for mechanical circulatory support and antibiotics, but she died $40 \mathrm{~h}$ after admission. Autopsy findings showed necrotic and suppurative myocardial changes due to group B Streptococcus (GBS). To date, only one case of bacterial myocarditis caused by GBS has been reported. We herein report a case of GBS myocarditis, the etiology of which is poorly understood due to the limited number of cases. Bacterial myocarditis should be considered in patients with sepsis and myocardial dysfunction.
\end{abstract}

Key words: bacterial myocarditis, biopsy, group B Streptococcus, sepsis

(Intern Med Advance Publication)

(DOI: 10.2169/internalmedicine.5498-20)

\section{Background}

Group B Streptococcus (Streptococcus agalactiae) (GBS) is a common cause of neonatal sepsis but can also affect immunocompromised adults (1) and healthy older individuals (2). The clinical presentation of GBS infection is variable, and only 1 case of bacterial myocarditis caused by GBS has been reported in the past 30 years (3). We herein report an autopsy case of suppurative and rapidly progressive myocarditis caused by GBS. We describe the clinical course and autopsy findings of this case.

\section{Case}

A 61-year-old Japanese woman with no significant medical history visited a neighboring clinic with the acute onset of a fever, nausea, diarrhea, and dull and persistent back pain. She was transferred to our hospital for a further evaluation. She was not taking any regular medications, and there was no history of alcohol abuse, food poisoning, or foreign travel.

Her body temperature, pulse rate, blood pressure, and respiratory rate were $36.4{ }^{\circ} \mathrm{C}, 98 \mathrm{bpm}$ (sinus rhythm), 91/59 $\mathrm{mmHg}$, and 23 breaths/min, respectively, and she was alert on admission. She complained of the same symptoms, but the pain was tolerable. Computed tomography of the chest and abdomen showed no infection. Electrocardiography (ECG) conducted on admission showed slight and nonspecific ST elevation in leads V1-3 (Fig. 1). A blood examination showed elevated liver enzyme, serum creatinine, Creactive protein, and high-sensitivity troponin I levels(Table 1).

A presumed diagnosis of hepatitis was made, and she was admitted to the general ward by the first examining physician for observation. Her symptoms worsened, and her consciousness level dropped $12 \mathrm{~h}$ after admission to our hospital. Repeat ECG showed further ST elevation (Fig. 1) and further elevation of liver enzyme, serum creatinine, and high sensitivity troponin I and lactate levels (Table 1). Based on these findings, rapidly progressing myocarditis was suspected.

\footnotetext{
${ }^{1}$ Graduate School of Medical Science, Kumamoto University, Japan, ${ }^{2}$ Department of Emergency and Critical care Medicine, Oita City Medical Association's Almeida Memorial Hospital, Japan, ${ }^{3}$ Department of Cardiovascular Medicine, Oita City Medical Association's Almeida Memorial Hospital,, Japan, ${ }^{4}$ Department of Laboratory Medicine, Oita City Medical Association's Almeida Memorial Hospital, Japan and ${ }^{5}$ Disaster Medical Education and Research Center, Kumamoto University, Japan

Received: June 3, 2020; Accepted: August 1, 2021; Advance Publication by J-STAGE: September 18, 2021

Correspondence to Dr. Ryuta Nakashima, hanazono0408@yahoo.co.jp
} 


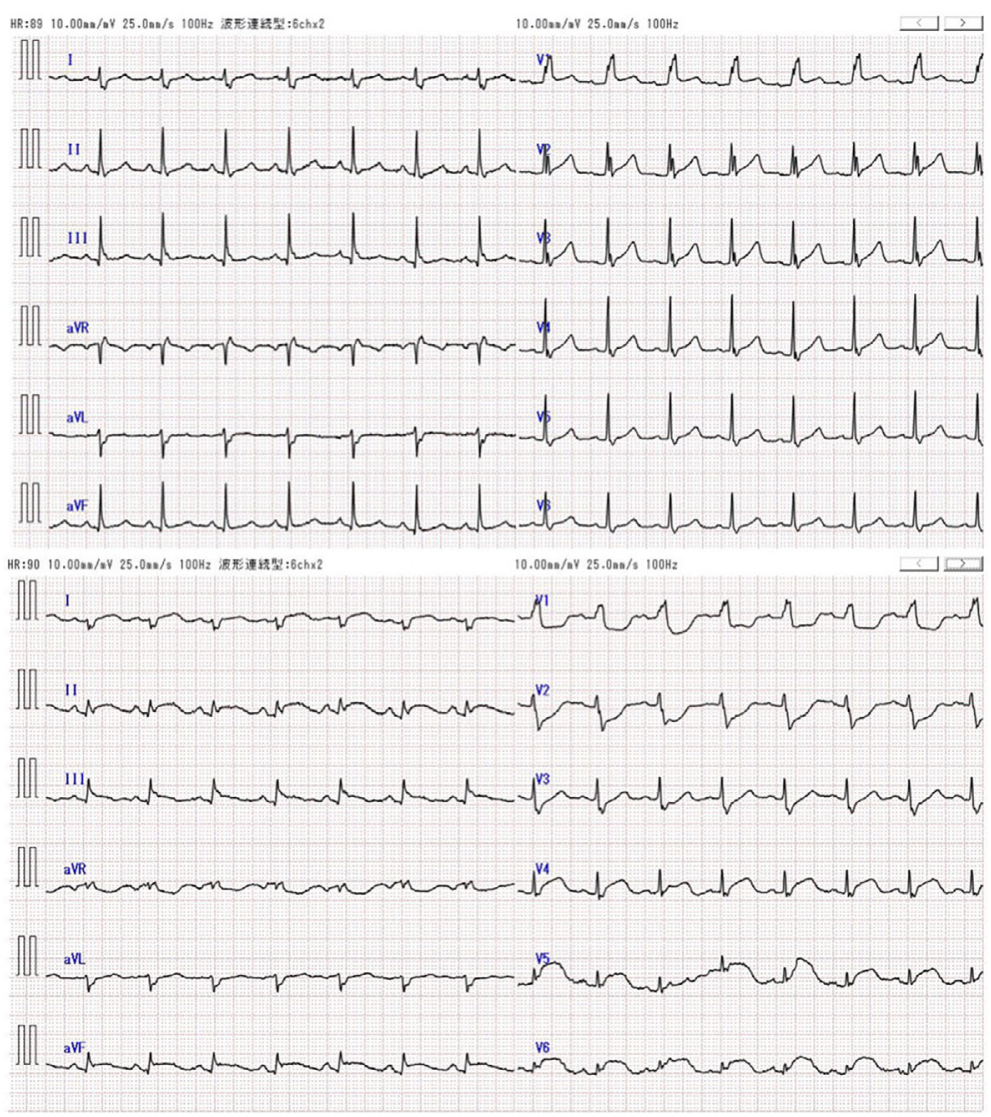

Figure 1. Findings of a 12-lead electrocardiogram on admission to our hospital (upper) and after deterioration (lower). Slight ST segment elevation was observed in leads V1-3 on admission, with a further elevation in leads II, III, $\mathrm{aV}_{\mathrm{F}}$, and V4-6 noted after deterioration.

Table 1. Laboratory Data on Admission to Our Hospital and after Deterioration.

\begin{tabular}{lrc}
\hline & on admission & \multicolumn{2}{c}{ after deterioration } \\
\hline Total birilubin & 1.3 & $1.9 \mathrm{mg} / \mathrm{dL}$ \\
Direct birilubin & 1.0 & $1.5 \mathrm{mg} / \mathrm{dL}$ \\
AST & 1,697 & $3,399 \mathrm{U} / \mathrm{L}$ \\
ALT & 291 & $638 \mathrm{U} / \mathrm{L}$ \\
LDH & 1,243 & $3,122 \mathrm{U} / \mathrm{L}$ \\
ALP & 252 & $227 \mathrm{U} / \mathrm{L}$ \\
$\gamma$ GTP & 272 & $255 \mathrm{U} / \mathrm{L}$ \\
CK & 71 & $11099 \mathrm{U} / \mathrm{L}$ \\
CK-MB & 1.7 & $1377 \mathrm{ng} / \mathrm{mL}$ \\
high-sensitivity troponin & 219.000 & $362,104.000 \mathrm{pg} / \mathrm{mL}$ \\
BUN & 16 & $26 \mathrm{mg} / \mathrm{dL}$ \\
Creatinine & 1.11 & $1.45 \mathrm{mg} / \mathrm{dL}$ \\
Na & 134 & $132 \mathrm{mEq} / \mathrm{L}$ \\
K & 4.2 & $4.6 \mathrm{mEq} / \mathrm{L}$ \\
Cl & 103 & $96 \mathrm{mEq} / \mathrm{L}$ \\
CRP & 5.53 & $10.63 \mathrm{mg} / \mathrm{dL}$ \\
WBC & 4,650 & 900 \\
RBC & 323 & $35810^{\wedge} 4 / \mu \mathrm{L}$ \\
hemoglobin & 9.3 & $10.4 \mathrm{~g} / \mathrm{dL}$ \\
platelet & 14.6 & $11.310^{\wedge} 4 / \mathrm{\mu L}$ \\
PT-INR & 1.26 & 1.56 \\
APTT & 29.6 & $\mathrm{~N} / \mathrm{A} \mathrm{sec}$ \\
Fibrinogen & 216 & $\mathrm{~N} / \mathrm{A} \mathrm{mg/dL}$ \\
D-dimer & 6.9 & $\mathrm{~N} / \mathrm{A} \mu \mathrm{gg} / \mathrm{mL}$ \\
pH & 7.418 & 7.397 \\
PaCO2 & 29.8 & $103.0 \mathrm{mmHg}$ \\
PaO2 & 69.7 & $12.9 \mathrm{mmHg}$ \\
HCO3 & 18.9 & $7.8 \mathrm{mmol} / \mathrm{L}$ \\
BE & -4.4 & $-15.5 \mathrm{mmol} / \mathrm{L}$ \\
lactate & 4.7 & $11.2 \mathrm{mmol} / \mathrm{L}$ \\
\hline & &
\end{tabular}

She underwent an endomyocardial biopsy, coronary angiography, and right heart catheterization $14 \mathrm{~h}$ after admission. Coronary angiography showed no abnormal findings, but the cardiac index was very low $\left(1.12 \mathrm{~L} / \mathrm{min} / \mathrm{m}^{2}\right)$ on right heart catheterization. She received mechanical ventilation, intraaortic balloon pumping (IABP), and veno-arterial extracorporeal membrane oxygenation (VA-ECMO) (Capiox ${ }^{\circledR}$; Terumo, Tokyo, Japan) before being transferred to the intensive-care unit (ICU).

Transthoracic echocardiography conducted in the ICU showed severe global hypokinesis with pericardial effusion (Fig. 2). She received massive fluid and blood products $(9,550 \mathrm{cc} / 24 \mathrm{~h}$, including $1,000 \mathrm{cc}$ of $5 \%$ albumin preparation, 12 units of red blood cells, 14 units of fresh-frozen plasma, and 10 units of platelet concentrate), vasopressin (up to $1 \mathrm{unit} / \mathrm{h}$ ), and dobutamine (up to $10 \mu \mathrm{g} / \mathrm{kg} / \mathrm{min}$ ). Continuous renal replacement therapy was initiated because of oliguria. Despite these interventions, her cardiac index measured by a pulmonary artery catheter (PAC) was below the measurable limit, and the flow rate on VA-ECMO could not be maintained above $1.0 \mathrm{~L} /$ minute. Chest radiography after these procedures showed pulmonary edema (Fig. 3).

Twenty hours after admission to our hospital, the results of the endomyocardial biopsy were obtained, showing a bacterial mass, degeneration of myocardial cells, and infiltration with inflammatory cells (Fig. 4). We immediately examined 


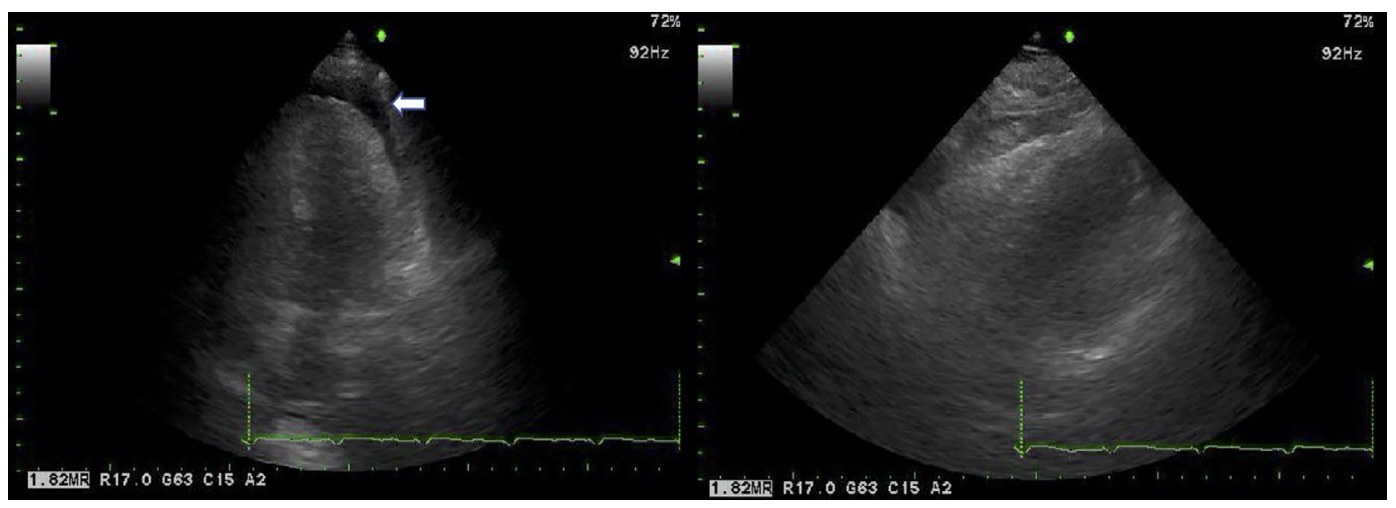

Figure 2. Transthoracic echocardiography (apical 2-chamber view, poor study) performed after admission to the ICU. The left ventricular wall motion was very slight, and the ejection fraction was not measurable. Pericardial effusion collection is marked with a white arrow.
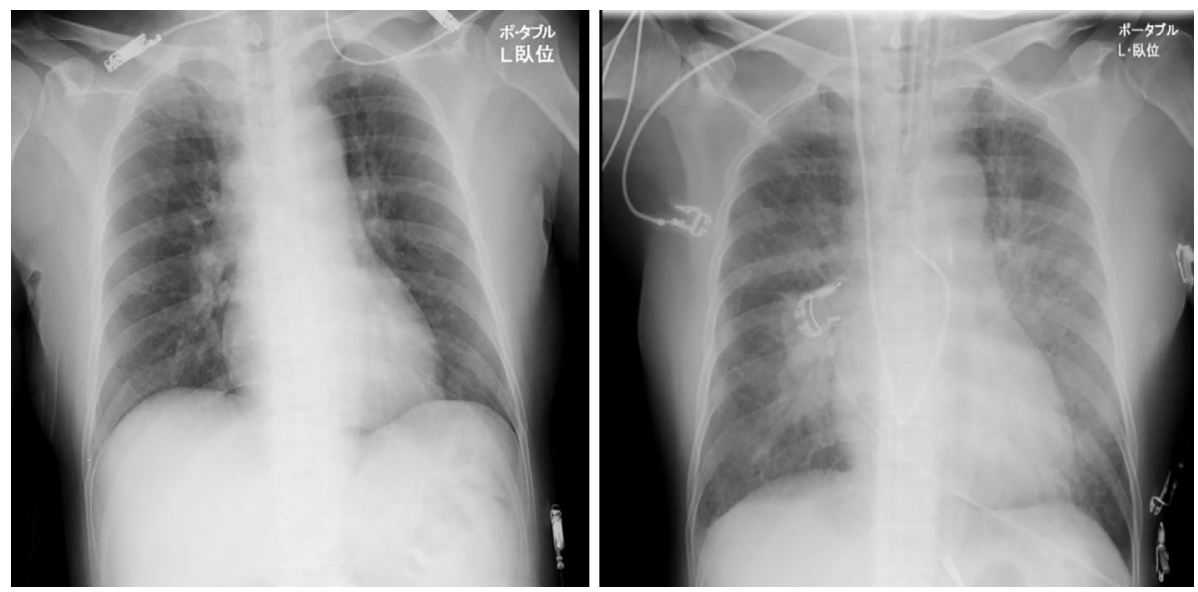

Figure 3. Chest radiography obtained after arrival at our hospital (left) and admission to the ICU (right).

her blood, urine, and sputum cultures and administered antibiotics (doripenem $3 \mathrm{~g} /$ day, linezolid 1,200 mg/day, and fosfluconazole $800 \mathrm{mg} /$ day), but her condition did not improve, and she ultimately died $40 \mathrm{~h}$ after admission to our hospital (Fig. 5).

An autopsy revealed bacterial infection around the necrotic cardiac tissue, without vegetation, deformation of valves, or tears in the endocardium. The bacteria were suggested to have spread around the necrotic tissue of the cardiac muscle. As the patient had no notable cardiac history, the necrotic tissue was thought to have formed during the infection.

An evaluation of the urinary tract revealed acute tubular necrosis and focal distributed microabscesses in the renal medulla, but no infectious changes were observed in the glomeruli, renal pelvis, or urinary bladder. Based on these findings, a retrograde urinary tract infection was ruled out. The liver showed necrotic changes around the central vein with low inflammatory cell infiltration and few necrotic changes around the portal vein. These findings indicated septic liver damage or congestive liver change, and there was no evidence of hepatitis (Fig. 4). Other organs, including the uterus, showed no infectious changes. GBS (serotype
III) was detected in the myocardial specimen and in blood and urine samples (Table 2). No organisms were detected in the PAC, IAB, ECMO, or central venous catheter. The cause of death was thus deemed to be bacterial myocarditis without a primary focus.

\section{Discussion}

We encountered a case of bacterial myocarditis caused by GBS that was confirmed by bacteriological and pathological methods.

GBS is a well-known pathogen that causes vertical infections in neonates. A recent study in Japan (4) indicated that invasive GBS disease has become an important problem among non-pregnant adults, especially the elderly, and those with diabetes or malignancy. In that study, the most common serotype of GBS was Ib (39.5\%), followed by $\mathrm{V}$ (16.0\%) and II (13.8\%). The most common clinical manifestation of GBS infection is bacteremia, without a primary focus. Endocarditis was observed in only 17 (3.8\%) of the 443 cases in that previous study. In our literature review of English articles, we identified only 1 case of bacterial myocarditis caused by GBS reported within the last 30 years (3). 


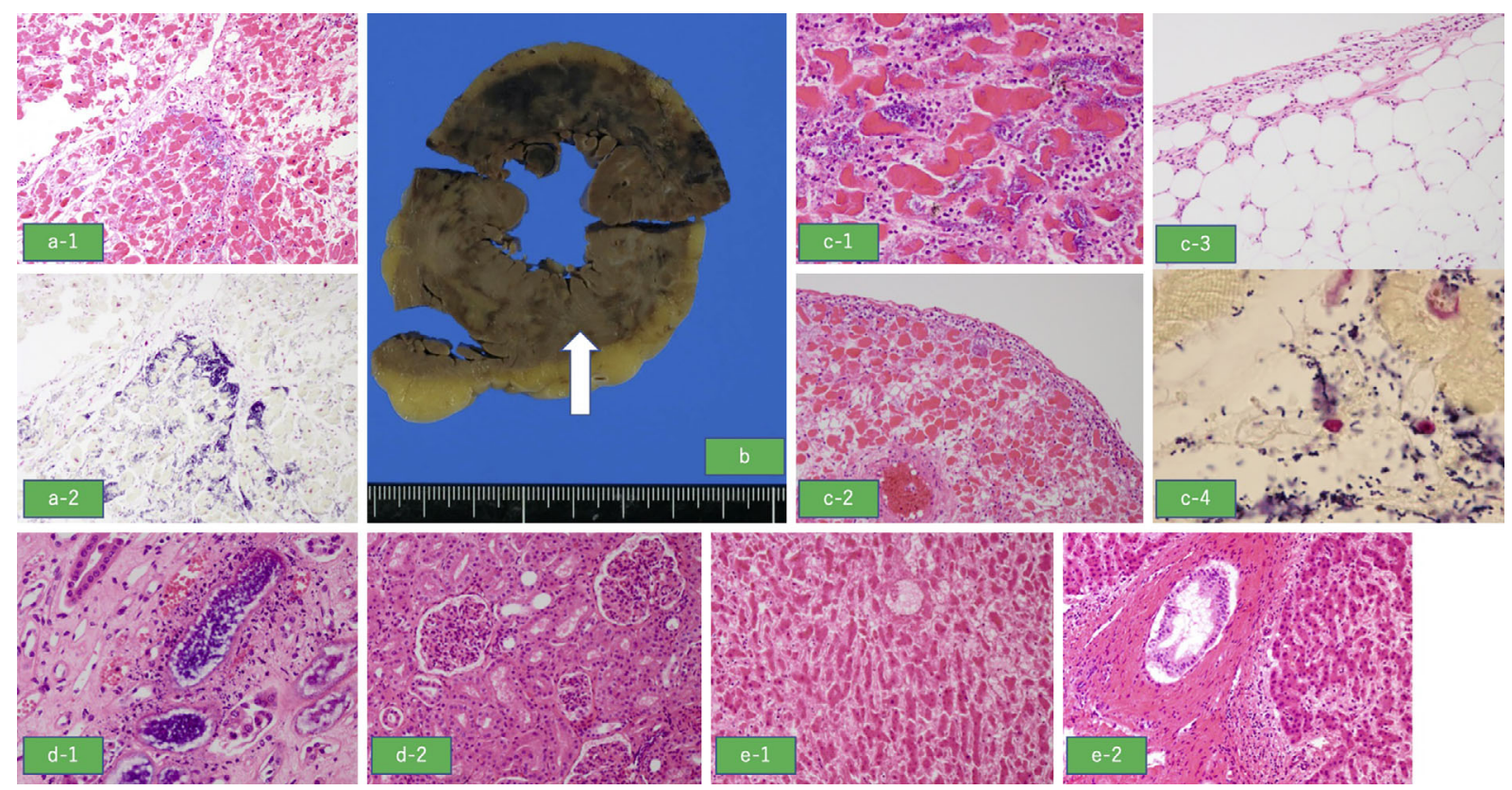

Figure 4. Findings of an endomyocardial biopsy. A specimen revealed bacteria and inflammatory cell infiltration with degeneration of myocardial cells [a-1: Hematoxylin and Eosin (H\&E) staining, a-2; Gram stain]. Macroscopic findings. The heart showed the presence of abscesses and necrosis (arrow) throughout the left ventricular wall (b). Histology findings of the autopsy. Necrotic cells and infiltrated inflammatory cells were detected in the cardiac muscle specimen (c-1; H\&E staining). The endomyocardium (c-2; H\&E staining) and epicardium (c-3; H\&E staining) were intact. Gram staining revealed the presence of Gram-positive bacteria (c-4). In the kidney, acute tubular necrosis and focal distributed microabscesses in the renal medulla were present (d-1; H\&E staining), but no infectious changes were observed in the glomerulus (d-2; H\&E staining). In the liver, necrotic changes were observed around the central vein with few inflammatory cells (e-1; H\&E staining), and few necrotic changes were observed around the portal vein (e-2; H\&E staining).

In our case, direct infection of the myocardium by GBS was confirmed bacteriologically and pathologically. The GBS in urinary specimens was thought to have been derived from the bloodstream, as there was no evidence of a primary focus on a pathological evaluation. The pathophysiology of the rapidly progressive myocardial GBS infection in this patient was unclear, as she was middle-aged and had no comorbidities. This is in keeping with the limited evidence regarding the etiology of bacterial myocarditis due to GBS.

There are two types of cardiac contraction dysfunction associated with sepsis: bacterial myocarditis and septic cardiomyopathy, which has recently been described in great detail. Bacterial myocarditis is rare and is a direct bacterial infection of the myocardium that usually presents as a localized abscess (5). Septic cardiomyopathy is characterized by left, right, or bi-ventricular dysfunction caused by changes in intracellular $\mathrm{Ca}^{2+}$ handling, mitochondrial dysfunction, and apoptosis of myocytes. These changes are regulated by cytokines, chemical species (e.g. reactive oxygen and nitrogen species), and lipid mediators (e.g. eicosanoids and prostaglandins) (6). Edematous changes in myocytes have been observed because of increased plasma membrane permeability in animal models of septic cardiomyopathy (7).

ECG changes in bacterial myocarditis include non-specific
ST-T wave changes, arrhythmias, or conduction abnormalities $(5,8)$. Owing to the limited number of published cases, the etiology of echocardiography in bacterial myocarditis is unknown (8), but echocardiography showed wall thickening and brightness in a previously reported case (9). In septic cardiomyopathy, the electrocardiography and echocardiography findings are conflicting (6).

Cardiac magnetic resonance imaging is more specific than ECG or echocardiography for detecting bacterial myocarditis $(6,10)$ but it is not appropriate for patients with shock because of safety issues. An endomyocardial biopsy is a more accurate method of identifying or differentiating cardiac dysfunction due to sepsis, but a biopsy is invasive, and the optimal timing for an endomyocardial biopsy is controversial (10).

Formal criteria for bacterial myocarditis and septic cardiomyopathy have not been established. In previously reported cases, the distinction between bacterial myocarditis and sepsis-induced myocardial dysfunction was unclear, although some cases were diagnosed by a biopsy or autopsy $(9,11,12)$.

Causes of acute myocarditis are varied, including viral, eosinophilic, lymphocytic, and immune checkpoint inhibitorassociated, and cases can progress rapidly. Ammirati et al. 


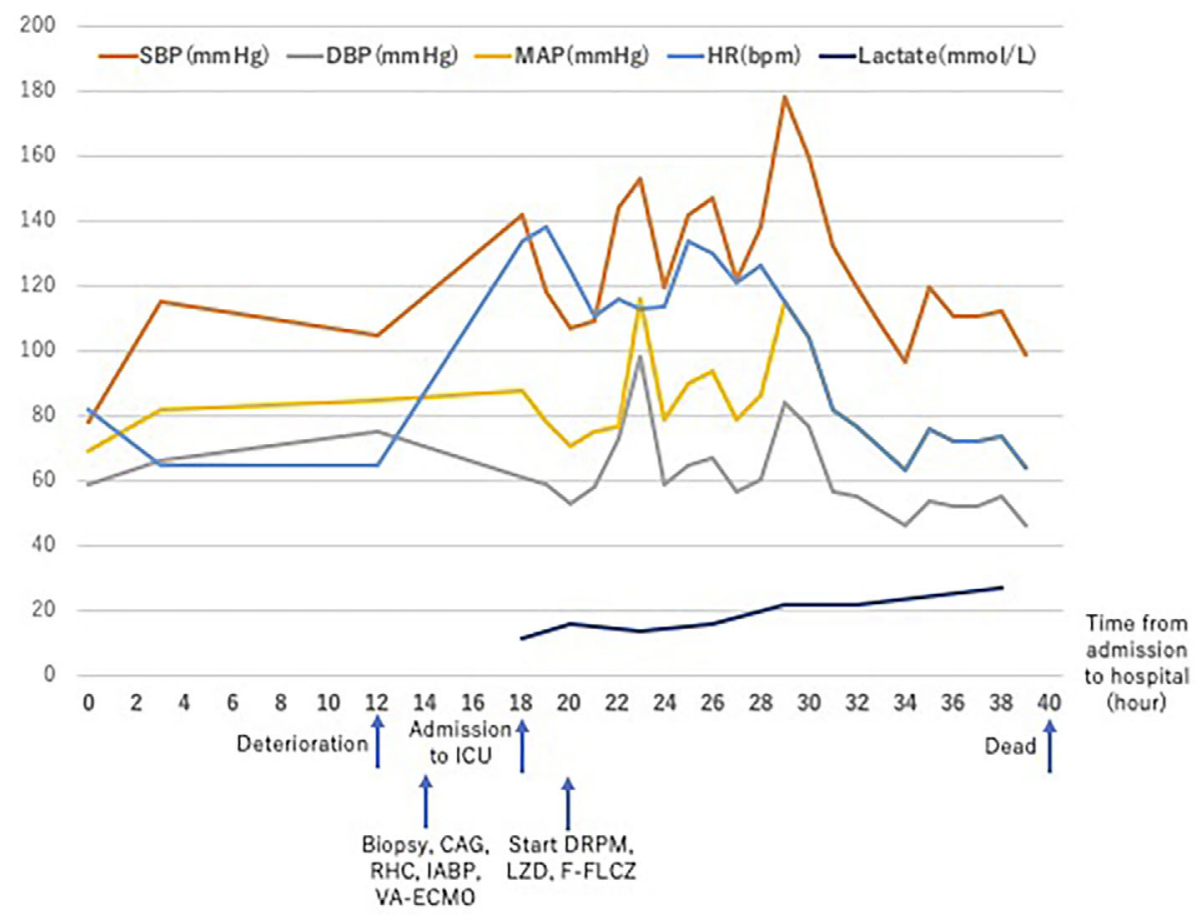

Figure 5. The patient's therapeutic course. SBP: systolic blood pressure, DBP: diastolic blood pressure, MAP: mean arterial pressure, HR: heart rate, CAG: coronary angiography, RHC: right heart catheterization, IABP: intra-aortic balloon pumping, VA-ECMO: veno-arterial extracorporeal membrane oxygenation, DRPM: doripenem, LZD: linezolid, F-FLCZ: fosfluconazole

Table 2. Drug Sensitivity of GBS.

\begin{tabular}{|c|c|c|c|c|c|c|}
\hline & & blood & & myocardium & urine & \\
\hline strain & & GBS & & GBS & GBS & \\
\hline \multirow[t]{18}{*}{ MIC } & PCG & & 0.06 & 0.06 & & 0.06 \\
\hline & $\mathrm{ABPC}$ & & 0.12 & 0.12 & & 0.12 \\
\hline & CTM & & $<=0.5$ & $<=0.5$ & & $<=0.5$ \\
\hline & CTRX & & $<=0.12$ & $<=0.12$ & & $<=0.12$ \\
\hline & CZOP & & $<=0.12$ & $<=0.12$ & & $<=0.12$ \\
\hline & CFPM & & $<=0.5$ & $<=0.5$ & & $<=0.5$ \\
\hline & CDTR-P & & $<=0.06$ & $<=0.06$ & & $<=0.06$ \\
\hline & MEPM & & $<=0.12$ & $<=0.12$ & & $<=0.12$ \\
\hline & CVA/AMPC & & $<=0.25$ & $<=0.25$ & & $<=0.25$ \\
\hline & EM & & 0.5 & 1 & & 1 \\
\hline & $\mathrm{AZM}$ & & 1 & 2 & ? & 2 \\
\hline & CLDM & & $<=0.12$ & $<=0.12$ & & $<=0.12$ \\
\hline & MINO & & $>4$ & $>4$ & & $>4$ \\
\hline & LVFX & & 0.5 & 0.5 & & 0.5 \\
\hline & VCM & & 0.5 & 0.5 & & 0.5 \\
\hline & $\mathrm{CP}$ & & $<=4$ & $<=4$ & & $<=4$ \\
\hline & ST & & $<=0.5$ & $<=0.5$ & & $<=0.5$ \\
\hline & RFP & & $<=1$ & $<=1$ & & $<=1$ \\
\hline
\end{tabular}

MIC: minimum inhibitory concentration, PCG: penicillin G, ABPC: ampicillin, CTM: cefotiam, CTRX: ceftriaxone, CZOP: cefozopran, CFPM: cefepime, CDTR-P: cefditoren pivoxil, MEPM: meropenem, CVA/AMPC: clavulanic acid/amoxicillin, EM: erythromycin, AZM: azithromycin, CLDM: clindamycin, MINO: minocycline, LVFX: levofloxacin, VCM: vancomycin, CP: chloramphenicol, ST: sulfamethoxazoletrimethoprim, RFP: rifampicin

advocated a risk-based approach stratified by symptoms, ECG, electrocardiography, biomarkers, and autoimmu- nity (13). Bacteriological and pathological diagnoses of bacterial myocarditis are important for understanding and confirming the epidemiology of bacterial myocarditis in patients with sepsis and myocardial dysfunction in accordance with the risk-based approach like that.

\section{Conclusion}

We encountered a case of fulminant suppurative bacterial myocarditis caused by GBS. Bacterial myocarditis should be considered in cases of sepsis with rapidly progressive myocardial dysfunction, and an endomyocardial biopsy should be considered for confirmation of the diagnosis.

The authors state that they have no Conflict of Interest (COI).

\section{References}

1. Rosa-Fraile M, Spellerberg B. Reliable Detection of Group B Streptococcus in the Clinical Laboratory. J Clin Microbiol 55: 2590-2598, 2017.

2. Edwards MS, Baker CJ. Group B streptococcal infections in elderly adults. Clin Infect Dis 41: 839-847, 2005.

3. Bateman AC, Richards M, Pallett AP. Fatal myocarditis associated with a Lancefield group B streptococcus. J Infect 36: 354-355, 1998.

4. Morozumi M, Wajima T, Takata M, Iwata S, Ubukata K. Molecular characteristics of Group B Streptococci isolated from adults with invasive infection in Japan. J Clin Microbiol 54: 2695-2700, 2016.

5. Wasi F, Shuter J. Primary bacterial infection of the myocardium. Front Biosci 8: s228-s231, 2003. 
6. Ehrman RR, Sullivan AN, Favot MJ, et al. Pathophysiology, echocardiographic evaluation, biomarker findings, and prognostic implications of septic cardiomyopathy: a review of the literature. Crit Care 22: 112, 2018.

7. Celes MR, Torres-Dueñas D, Malvestio LM, et al. Disruption of sarcolemmal dystrophin and beta-dystroglycan may be a potential mechanism for myocardial dysfunction in severe sepsis. Lab Invest 90: 531-542, 2010.

8. Shirani J, Ilercil A, Chandra M, Jelicks LA, Tanowitz HB. Cardiovascular imaging in clinical and experimental acute infectious myocarditis. Front Biosci 8: e323-s336, 2003.

9. Elias T, Roberts I, Jones N, Sabharwal N, Leeson P. Suppurative bacterial myocarditis: echocardiographic and pathological findings. Eur Heart J 29: 489, 2007.

10. Fung G, Luo H, Qiu Y, Yang D, McManus B. Myocarditis. Circ
Res 118: 496-514, 2016.

11. LeLeiko RM, Bower DJ, Larsen CP. MRSA-associated bacterial myocarditis causing ruptured ventricle and tamponade. Cardiology 111: 188-190, 2008.

12. Komuro J, Ueda K, Kaneko M, Nitta S, Kasao M, Yokoyama M. Various Cardiac Abnormalities Caused by Bacterial Myocarditis. Int Heart J 59: 229-232, 2018.

13. Ammirati E, Veronese G, Bottironi M, et al. Update on acute myocarditis. Trends Cardiovasc Med S1050-1738(20)30079-7.

The Internal Medicine is an Open Access journal distributed under the Creative Commons Attribution-NonCommercial-NoDerivatives 4.0 International License. To view the details of this license, please visit (https://creativecommons.org/licenses/ by-nc-nd/4.0/).

\section{(C) The Japanese Society of Internal Medicine Intern Med Advance Publication}

